\title{
HPLC-ESI-MS/MS ANALYSIS OF OXIDIZED DI-CAFFEOYLQUINIC ACIDS GENERATED BY METALLOPORPHYRIN-CATALYZED REACTIONS
}

\author{
Michel D. Santos and Norberto P. Lopes* \\ Departamento de Física e Química, Faculdade de Ciências Farmacêuticas de Ribeirão Preto, Universidade de São Paulo, Via do \\ Café, s/n, 14040-903 Ribeirão Preto - SP, Brazil \\ Yassuko Iamamoto \\ Departamento de Química, Faculdade de Filosofia Ciências e Letras de Ribeirão Preto, Universidade de São Paulo, Av. \\ Bandeirantes, 3900, 14040-901 Ribeirão Preto - SP, Brazil
}

Recebido em 19/12/07; aceito em 7/4/08; publicado na web em 29/4/08

\begin{abstract}
This paper reports an HPLC-ESI-MS/MS investigation on the oxidation of 3,5- and 4,5- dicaffeoylquinic acid using iron(III) tetraphenylporphyrin chloride as catalyst. Two major mono-oxidised products of the quinic acid moiety have been identified for both compounds. However, only the 4,5-derivative afforded two different tri-oxo products. Thus, it seems that the oxidation pattern depends on the number and positions of the caffeic acid moieties present in caffeoylquinic acid molecules.
\end{abstract}

Keywords: metalloporphyrin biomimetic reaction; mass spectrometry; di-caffeoylquinic acids.

\section{INTRODUCTION}

Caffeoylquinic acids (CAs) are non-flavonoid catecholic compounds widely distributed in nature. There has been an increasing interest in the biological role of these compounds due to their occurrence in foods ${ }^{1-3}$ and in medicinal plants. ${ }^{4-6}$ Several reports in the literature show that CAs present a vast array of biological activities, such as antioxidant, ${ }^{7,8}$ analgesic, ${ }^{9}$ antimutagenic ${ }^{10}$ and immunomodulatory. ${ }^{11}$ Due to the increasing importance of natural products in human health and World trade, parameters such as quality, safety and efficacy have become key issues. ${ }^{12}$ In this sense, besides characterizing the biological activities, it is extremely important to evaluate the pharmacokinetic parameters (absorption, distribution, metabolism, and excretion) of these compounds in early stages of the drug development process. ${ }^{13}$

CAs are characterized by the presence of chromophore moieties (with one to four aromatic rings) in their structure. Therefore, this class of compound allows the application of liquid chromatography with ultraviolet detection (HPLC-ESI-UV) for both identification and quantification procedures. However, as previously described for other conjugated polyenes, the analysis of such compounds requires complete chromatographic resolution, which in some cases is not achievable. ${ }^{14}$ More recently, ESI-MS/MS has been employed to analyze natural products with conjugated systems, combining structure elucidation and quantification. ${ }^{15,16}$ An HPLC-ESI-MS investigation of 5-caffeoylquinic acid (5-CA) in human plasma and urine showed a good sensitivity for this compound after a post-column reaction, using negative mode ESI. ${ }^{17} \mathrm{~A}$ systematic investigation carried out by Miketova and co-workers showed that ESI-MS analyses of CAs afforded considerable structural information in both modes of operation. ${ }^{18}$ Therefore, we performed a comparative ESI-MS (negative and positive mode) analysis of 5-CA, resulting in an unexpected increased number of ions in the positive mode than in the negative mode when no postcolumn reagent is added. ${ }^{19}$ These results confirm that it is feasible to perform analyses in both ionisation modes for metabolomic, absorption, distribution or excretion (toxicological) studies.

*e-mail: npelopes@fcfrp.usp.br
There is relatively little information in the literature regarding metabolism, excretion and enzymatic oxidation of CAs. ${ }^{2,19}$ Most of the studies normally employ commercial samples of 5-CA (a monossubstituted caffeoylquinic acid) or its constituents (caffeic and quinic acids and related molecules) for the examination of phase 2 metabolism. Just recently, a comparative study was carried out to evaluate the biomimetic oxidation of 5-CA employing an in vitro system of drug metabolism. ${ }^{19}$ Cytochrome P450 (CYP) enzymes, which are mostly present in the liver of mammals, are responsible for the oxygenative and oxidative metabolism (phase 1) of drugs and xenobiotics. ${ }^{20}$ However, several problems are associated with the use of in vivo systems in studying drug metabolism, such as the small quantities of the metabolites produced, the difficulty of their isolation from the biological matrices and the variable potency of in vitro liver slice preparations. ${ }^{21}$ In this sense, metalloporphyrins represent an alternative approach as biomimetic catalysts to study the behavior of biologically active compounds under oxidative conditions. ${ }^{21-24}$ Investigation of 5-CA oxidation by iron(III) tetraphenylporphyrin chloride afforded several oxidation products that were detected and identified by sequential MS analyses. ${ }^{19}$ These data, in comparison to an in vitro metabolism assay employing isolated rat liver mitochondria, confirm that the major oxidised product obtained from mitochondrial metabolism corresponds to the major product formed by the biomimetic (metalloporphyrin) system, confirming the validity of the use of metalloporphyrin-catalysed oxidation studies of other non-flavonoid catecholic compounds, such as dissubstituted caffeoylquinic acids.

The goal of this study is, therefore, to isolate from a Brazilian medicinal plant the compounds 3,5-di-caffeoylquinic acid (3,5DCA) and 4,5-di-caffeoylquinic acid (4,5-DCA), submit them to biomimetic oxidation reactions catalysed by metalloporphyrin and analyze the oxidised products by HPLC-ESI-MS/MS.

\section{EXPERIMENTAL}

\section{Materials}

For the preparation, processing and analysis of the plant extract and fractions we employed commercial grade (J.T. Baker) or HPLC 
grade (EM Science) solvents. For the metalloporphyrin oxidation reactions, iodosylbenzene ( $\mathrm{PhIO}$ ) was synthesised as previously described. ${ }^{25}$ Iron(III) tetraphenylporphyrin chloride $[\mathrm{Fe}(\mathrm{TPP})] \mathrm{Cl}$ (purchased from Midcentury), despite being a first generation catalyst, was chosen in spite of the previous results obtained by our group. ${ }^{19}$ Lychnophora ericoides (Asteraceae) was collected at Delfinópolis, Minas Gerais state, Brazil. ${ }^{9}$ The material was identified by Prof. João Semir, University of Campinas, where a voucher specimen was deposited (NPL-123; herbarium UEC).

\section{Phytochemical procedures}

The methanolic crude extract and fractions were obtained as previously described. ${ }^{9}$ The $n$ - $\mathrm{BuOH}$ fraction was cleaned-up (by ressuspending it in $\mathrm{MeOH}$, centrifuging and discarding the precipitate) and applied to a Sephadex LH-20 (400 g, Sigma) column using distilled $\mathrm{MeOH}$ as eluent. Fractions 11 and 12 were submitted to ${ }^{1} \mathrm{H}-$ NMR and ${ }^{13} \mathrm{C}-\mathrm{NMR}$ (300 and $75 \mathrm{MHz}$, respectively; Bruker) and ESI-MS. The data obtained were compared to literature values, ${ }^{18,26,27}$ and the compounds were identified as 3,5-di- $O-[E]$-caffeoylquinic acid (3,5-DCA; fraction $11,54.5 \mathrm{mg})$ and 4,5-di- $O$-[E]-caffeoylquinic acid (4,5-DCA; fraction 12, $45.0 \mathrm{mg}$ ) (Figure 1).
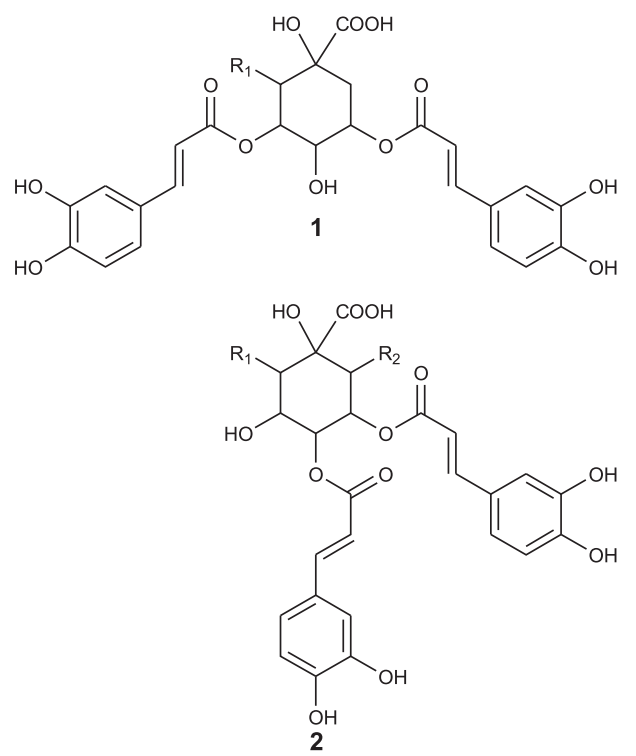

Figure 1. Structures of 3,5-di-O-[E]-caffeoylquinic acid $\left(1, R_{1}=H\right)$, 4,5-di$O$-[E]-caffeoylquinic acid $\left(2, R_{1}=R_{2}=H\right)$ and of their mono-oxidative products: mono-hydroxylated 3,5-DCA $\left(1, R_{1}=O H\right)$, oxo-3,5-DCA $\left(1, R_{1}=O\right)$, monohydroxylated 4,5-DCA $\left(2, R_{1}\right.$ or $\left.R_{2}=O H\right)$ and oxo-4,5-DCA $\left(2, R_{1}\right.$ or $\left.R_{2}=O\right)$

\section{Metalloporphyryn oxidation procedure}

The reaction $(2 \mathrm{~mL})$ in $\mathrm{MeCN}: \mathrm{H}_{2} \mathrm{O} 9: 1$ contained $30.0 \mathrm{mM}$ substrate (3,5-DCA or 4,5-DCA), $0.3 \mathrm{mM}$ [Fe(TPP)]Cl and 12.0 $\mathrm{mM} \mathrm{PhIO}$. All experiments were carried out at room temperature, in air, in a glass vessel equipped with a magnetic stirring bar. Reaction times were measured after the addition of $\mathrm{PhIO}$. At regular intervals, the magnetic stirring was stopped, an aliquot of mixture reaction $(20 \mu \mathrm{L})$ was withdrawn and added to $100 \mu \mathrm{L}$ of $\mathrm{MeOH}$. After porphyrin extraction, it was analysed by HPLC-ESI-MS/MS. Porphyrin extractions were carried out using C-18 mini-columns (Alltech) as previously reported. ${ }^{19}$

\section{HPLC, MS and HPLC-ESI-MS/MS conditions}

A Shimadzu HPLC system consisting of two LC-10AD solvent pumps, an SCL-10A system controller, a CTO-10AS column oven and a 7125 Rheodyne injector with a $20 \mu \mathrm{L}$ loop was used. Separations were carried out at $24{ }^{\circ} \mathrm{C}$ on a ShimPack ODS-18 column $(250 \mathrm{~mm} \times 4.6 \mathrm{~mm}, 5 \mu \mathrm{m}$, Shimadzu, Japan $)$ and a Lichrospher 100 pre-column ( $4.0 \mathrm{~mm} \times 4.0 \mathrm{~mm}, 5 \mu \mathrm{m}$, Merck). Elution was carried out at a flow-rate of $1.0 \mathrm{~mL} / \mathrm{min}$ using a $2 \%$ acetic acid in both $\mathrm{H}_{2} \mathrm{O}$ (solvent $\mathrm{A}$ ) and $\mathrm{MeOH}$ (solvent $\mathrm{B}$ ) as the mobile phase. Elution gradient: $0.0 \mathrm{~min}, 25 \% \mathrm{~B} ; 15 \mathrm{~min}, 100 \% \mathrm{~B}$; $17 \mathrm{~min}, 100 \% \mathrm{~B} ; 23 \mathrm{~min}, 15 \% \mathrm{~B} ; 30 \mathrm{~min}, 15 \% \mathrm{~B}$. A Micromass Quattro LC triple-stage quadrupole mass spectrometer, fitted with a Z-electrospray interface was used for all MS, MS-MS and HPLCESI-MS-MS experiments. A Valco valve was used to split the HPLC eluent and a flow rate of approximately $0.1 \mathrm{~mL} / \mathrm{min}$ was introduced into the source. The first mass analyzer was programmed to select each precursor ion as it eluted from the LC column. The second mass analyzer detected product ions produced by collision-induced dissociation of each parent ion. The most abundant product ion was selected for multiple-reaction monitoring (MRM). For the initial ESI-MS/MS analysis, solutions of 3,5-DCA and 4,5-DCA $(0.1 \mathrm{mg} / \mathrm{mL})$ were prepared in $\mathrm{MeOH}: \mathrm{H}_{2} \mathrm{O}$ with $2 \%$ acetic acid and infused into the ESI source at $5 \mu \mathrm{L} / \mathrm{min}$, using a syringe pump (model 1746, Harvard Apparatus).

\section{RESULTS AND DISCUSSION}

The phytochemical procedure afforded the compounds 3,5-di$\mathrm{O}$-[E]-caffeoylquinic acid (3,5-DCA) and 4,5-di-O-[E]-caffeoylquinic acid (4,5-DCA) at sufficient amount to allow the ESI-MS/MS and biomimetic oxidation studies. The MS spectra from both compounds showed the protonated molecule in addition to the cationised molecules. As expected, the MS/MS analysis showed the major fragment at $\mathrm{m} / \mathrm{z}, 163$, which originates from a neutral elimination of the quinic acid moiety (Figure 2). Detailed analysis shows that the 4,5-DCA seems to lose water more easily than 3,5-DCA since the ion at $\mathrm{m} / \mathrm{z}, 499$ is more prominent in the spectrum of 4,5-DCA than that of 3,5-DCA (Figure 2). The ions at $\mathrm{m} / \mathrm{z}, 337$ are produced by neutral elimination of the caffeic acid moiety, and further elimination of water affords the ion at $\mathrm{m} / \mathrm{z} 319$ (Figure 2). These two ions may be important for structure elucidation; however, they do not occur at high intensities enough for HPLC-ESI-MS/MS (MRM) studies.

MS analysis of the metalloporphyrin-catalyzed oxidation reactions of 3,5-DCA and 4,5-DCA showed, initially, two major signals for protonated ion species at $\mathrm{m} / \mathrm{z}, 531$ (oxo product, chemical structure is shown in Figure 1) and $\mathrm{m} / z .533$ (hydroxylated product, chemical structure is shown in Figure 1), in addition to the corresponding sodiated molecule of each compound that were also present at lower intensities. The MS/MS spectra of these major oxidation products $(\mathrm{m} / \mathrm{z}, 531$ and $\mathrm{m} / \mathrm{z}, 533)$ exhibit the single intense ion at $\mathrm{m} / \mathrm{z} 163$, confirming that there was no oxidation at the caffeic acid portion. Thus, the mono-oxidations (either hydroxylation or oxo formation) must occur in a similar way to that of our previous report on 5-CA biomimetic oxidations and liver (mitochondria) metabolism oxidation. ${ }^{19}$

The difference observed between the biomimetic oxidation of 3,5-DCA and 4,5-DCA was the presence of a minor 4,5-DCAtri-oxo product $(\mathrm{m} / \mathrm{z}, 543)$. In the previous 5 -CA study, ${ }^{19}$ the biomimetic oxidation of 5-CA yielded, preferentially, the monooxo product and similar amounts of the minor hydroxylated products (mono-oxidized) and of the di-oxo product, but no trioxo product was observed. Therefore, the very low amount of the tri-oxidized 4,5-DCA products and also the absence of the 3,5DCA-tri-oxo species may indicate, at least, the influence of the position of substitution on these oxidation reactions. To confirm this, samples collected from the oxidation reaction medium at 24 

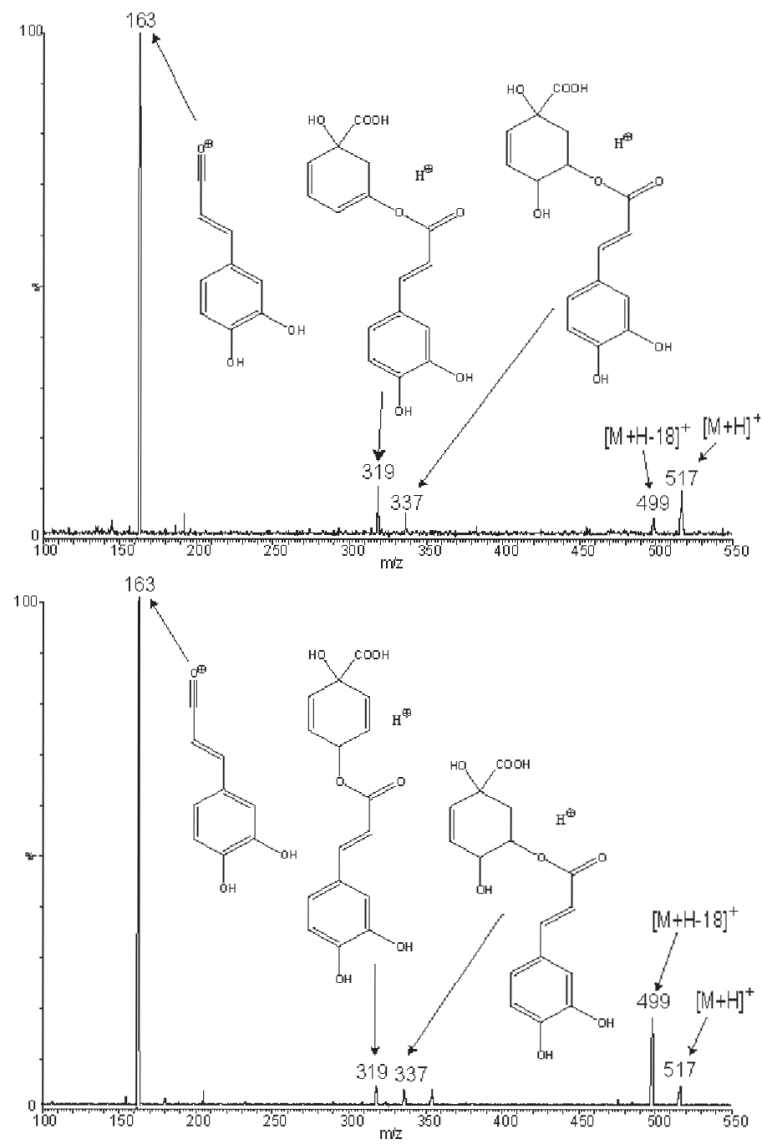

Figure 2. ESI-MS/MS spectra of 3,5-di-O-[E]-caffeoylquinic acid (upper panel) and 4,5-di-O-[E]-caffeoylquinic acid (lower panel)

h were analyzed by HPLC-ESI-MS/MS (MRM). The channels in the MRM mode, related to all possible combinations of 3,5-DCA and 4,5-DCA oxidized molecules, were selected. Figure 3 (a to d) shows representative MRM spectra of four different products for 4,5-DCA. This behavior was also observed in the analysis of 3,5-DCA oxidation reactions, in which the product ion $(\mathrm{m} / \mathrm{z}, 163)$ presents similar intensities to that of 4,5-DCA. However, only 4,5-DCA produced a signal at $\mathrm{m} / \mathrm{z} .543$ (product at $\mathrm{m} / \mathrm{z}, 163$ ) corresponding to the tri-oxo product probably generated by an extensive oxidation of the quinic acid moiety (oxo formation in the 2 and 6 methylenic positions and oxidation of the hydroxyl at position 3). Analysis of the retention times revealed that almost all oxidation products eluted after or similar to the precursors 3,5-DCA and 4,5-DCA $(\mathrm{m} / \mathrm{z}, 517$, Figure $3 \mathrm{~d})$. These results are expected by the formation of intra-molecular hydrogen bonding between the carboxylic acid hydrogen and the oxygen(s) at position 2 or 6 , as previously discussed and observed for 5-CA metabolites. ${ }^{19}$ Moreover, two different products were observed in the MRM chromatogram of the tri-oxo species $(\mathrm{m} / \mathrm{z}, 543$, Figure 3 a), one with the same previous chromatographic behavior (peak at $13.67 \mathrm{~min})$ and the other with a lower $t_{\mathrm{R}}(12.85 \mathrm{~min})$. The different $t_{\mathrm{R}}$ of all compounds were not significant, but may indicate that one of the compounds can be oxidized in an unexpected position (i.e., other than in the quinic acid portion). Also, the HPLC-ESI-TIC spectrum suggests the presence of a different ion species related to the compound eluting at 12.85 min that does not present the same oxidation pattern. To confirm this hypothesis, a new reaction mixture was analyzed by ESI-MS/MS direct infusion. Again, only 4,5-DCA showed the ion at $\mathrm{m} / \mathrm{z}, 543$, but the product ion spectrum exhibits a different profile (Figure 4). As expected, the minor ions produced by neutral elimination of caffeic acid were not observed since oxo formation in the quinic acid moiety reduces the number of $\alpha-\mathrm{H}$ available for the neutral elimination, as shown in Figure 2. In Figure 4, the ion at $m / z, 163$ is still the most intense ion, but a second ion at $\mathrm{m} / \mathrm{z} 177$ (related to the minor compound eluting at $12.85 \mathrm{~min}$, Figure 3 ) confirms the presence of oxidation at the caffeic acid moiety (formation of a $o$-quinone), in opposition to the previous observed results for 5CA. The literature provides examples of metalloporphyrincatalysed oxidations of aromatic substrates, including the formation of quinones. ${ }^{28-30}$ Although belonging to a series of position isomers, previous and present data indicate that the oxidation chemistry of 5-CA, 3,5-DCA and 4,5-DCA may vary, depending on their chemical structures. As already mentioned, CAs present a wide range of biological activities. However, the quality and extension of such activities seem to be dependent not only on the number of caffeic acid moieties but also on the position(s) of esterification(s) in the quinic portion. For example, 5-CA was shown to be ineffective in inhibiting the production of PGE $_{2}$ (a inflammatory mediator) by LPS-stimulated cultured cells. ${ }^{31}$ On the other hand, 3,5-DCA and 4,5-DCA showed, at low but not at high concentrations, inhibition of $\mathrm{PGE}_{2}$ in a similar model. Moreover, 4,5-DCA is more potent than 3,5-DCA in inhibiting the synthesis/release of MCP-3, a chemokine involved in the migration of inflammatory cells. In this research, we observed a common behavior for the major oxidized ions produced by biomimetic oxidation reactions of 4,5-DCA, 3,5-DCA and 5CA. On the other hand, analysis of the minor compounds indicates significant differences relating to the oxidation chemistry of these isomers, in a similar way to the variations in their biological activities.
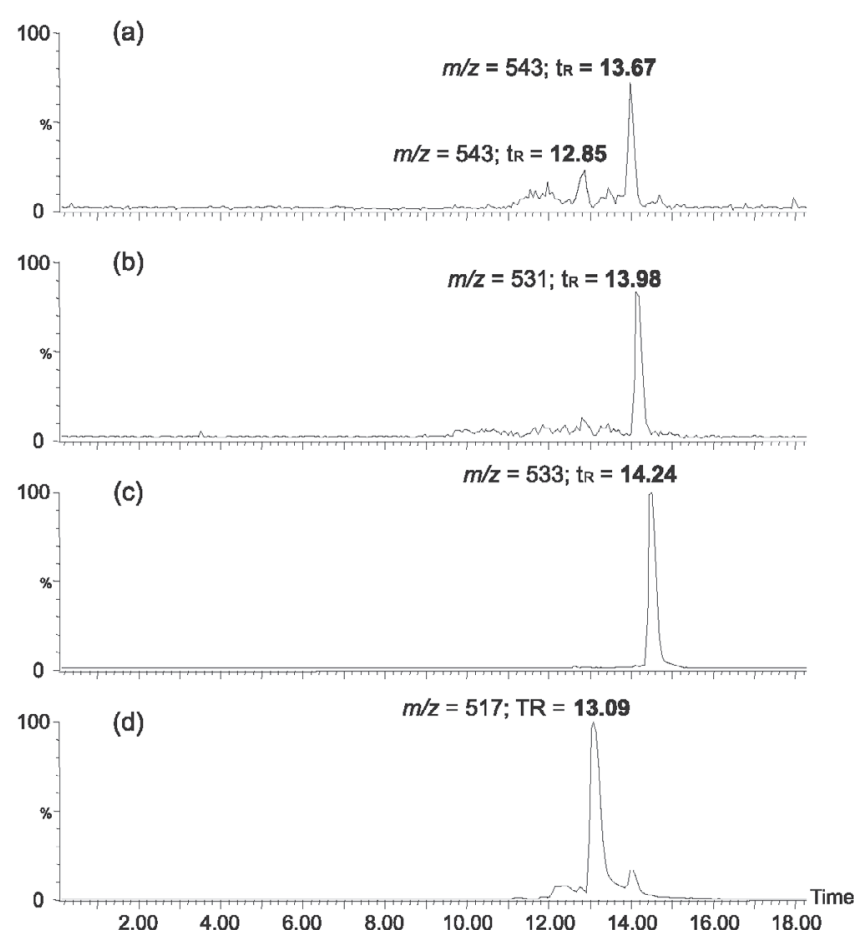

Figure 3. HPLC-ESI-MS-MS (MRM) chromatogram of 4,5-DCA oxidation reaction catalyzed by $\mathrm{Fe}(\mathrm{TPP}) \mathrm{Cl}$. (a) tri-oxo product, $\mathrm{m} / \mathrm{z}$.543; (b) oxo product, $m / z 531\left(R_{1}\right.$ or $\left.R_{2}=C=O\right)$; (c) hydroxylated product, $m / z 533\left(R_{1}\right.$ or $R_{2}=$ -OH); (d) 4,5-DCA, $\mathrm{m} / \mathrm{z} 517\left(R_{1}\right.$ or $\left.R_{2}=H\right)$. Conditions: column $(250 \mathrm{~mm} \times$ $4.6 \mathrm{~mm}, 5 \mu \mathrm{m}$ ); step gradient of $\mathrm{MeOH} / \mathrm{H}_{2} \mathrm{O}$ (containing $2 \%$ acetic acid); Elution gradient: $0.0 \mathrm{~min}, 25 \% \mathrm{~B} ; 15 \mathrm{~min}, 100 \% \mathrm{~B} ; 17 \mathrm{~min}, 100 \% \mathrm{~B} ; 23 \mathrm{~min}$, $15 \% \mathrm{~B} ; 30 \mathrm{~min}, 15 \% \mathrm{~B}$ 


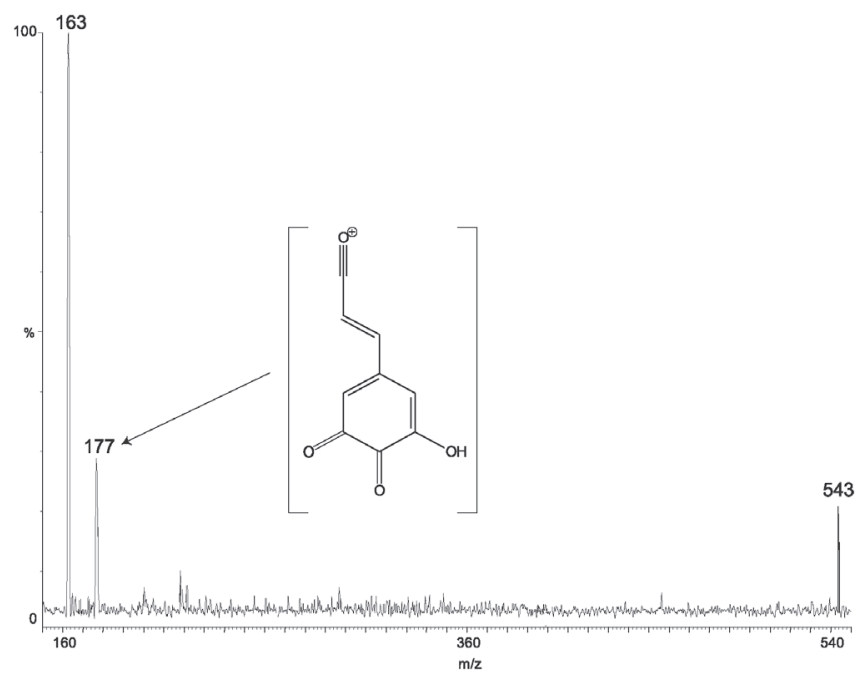

Figure 4. ESI-MS/MS spectrum of tri-oxo 4,5-DCA ( $\mathrm{m} / \mathrm{z}, 543)$, evidencing the product ion at $\mathrm{m} / \mathrm{z} 177$ (quinone formation in the caffeic acid portion)

\section{CONCLUSIONS}

In conclusion, the data presented here indicates that the oxidation of 3,5-DCA and 4,5-DCA catalyzed by metalloporphyrin (a system that mimics the reactions of CYP) served as a chemical model of phase 1 metabolism since the major products formed in these reactions are the mono-oxidized (hydroxylated and oxo) products, as previously reported for 5-CA oxidized products obtained by in vitro metabolism by isolated rat liver mitochondria. ${ }^{19}$ In addition, the selective oxidation of 4,5-DCA at the caffeic moiety, affording a quinonic structure, indicates that the biomimetic reaction may be affected by the substitution patterns of the molecules, a behavior which seems to be related to the different biological activities (quality and potency) of different isomers.

\section{ACKNOWLEDGEMENTS}

The authors acknowledge FAPESP, CAPES and CNPq (Instituto do Milênio) for financial support and fellowships.

\section{REFERENCES}

1. Laranjinha, J. A.; Almeida, L. M.; Madeira, V. M.; Biochem. Pharmacol. 1994, $48,487$.

2. Moridani, M. Y.; Scobie, H.; Jamshidzadeh, A.; Salehi, P.; O’Brien, J.; Drug Metab. Dispos. 2001, 29, 1432.

3. Clifford, M. N.; J. Sci. Food Agric. 1999, 79, 362

4. Gobbo-Neto, L.; Santos, M. D.; Kanashiro, A.; Almeida, M. C.; LucisanoValim, Y. M.; Lopes, J. L. C.; Souza, G. E. P.; Lopes, N. P.; Planta Med. 2005, 71, 3; Gobbo-Neto, L.; Lopes, N. P.; Quim. Nova 2007, 30, 374.

5. Li, Y. L.; But, P. P. H.; Ooi, V. E. C.; Antiviral Res. 2005, 68, 1.

6. Bilia, A. R.; Bergonzi, M. C.; Mazzi, V; Vincieri, F. F.; Drug Dev. Ind. Pharm. 2002, 28, 609 .

7. Zang, L.; Cosma, G.; Gardner, H.; Castranova, V.; Vallyathan, V.; Mol. Cell. Biochem. 2003, 247, 205.

8. Kono, Y.; Kobayashi, K.; Tagawa, S.; Adachi, K.; Ueda, A.; Sawa, Y.; Shibata, H.; Biochim. Biophys. Acta 1997, 1335, 335.

9. Santos, M. D.; Gobbo-Neto, L.; Albarella, L.; Souza, G. E. P.; Lopes, N. P.; J. Ethnopharmacol. 2005, 96, 545.

10. Yoshimoto, M.; Yahara, S.; Okuno, S.; Islam, M. S.; Ishiguro, K.; Yamakawa, O.; Biosci. Biotechnol. Biochem. 2002, 66, 2336.

11. Chiang, L.; Ng, L. T.; Chiang, W.; Lin, C.; Planta Med. 2003, 69, 600.

12. Mahady, G. B.; J. Nutr. 2001, 131, 1120 S.

13. Kostiainen, R.; Kotiaho, T.; Kuuranne, T.; Auriola, S.; J. Mass Spectrom. 2003, 38, 357.

14. Guaratini, T.; Vessecchi, R. L.; Lavarda, F. C.; Campos, P. M. B. G. M.; Naal, Z.; Gates, P. J.; Lopes, N. P.; Analyst 2004, 129, 1223.

15. Guaratini, T.; Vessecchi, R.; Pinto, E.; Colepicolo, P.; Lopes, N. P.; J. Mass Spectrom. 2005, 40, 963.

16. Crotti, A. E. M.; Vessecchi, R. L.; Lopes, J. L. C.; Lopes, N. P.; Quim. Nova 2006, 29, 287.

17. Cremin, P.; Kasim-Karakas, S.; Waterhouse, A. L.; J. Agric. Food Chem. 2001, 49, 1747.

18. Miketova, P.; Schram, K. H.; Whitney, J.; Kearns, E. H.; Timmermann, B. N.; J. Mass Spectrom. 1999, 34, 1240.

19. Santos, M. D.; Martins, P. R.; Santos, P. A.; Bortocan, R.; Iamamoto, Y.; Lopes, N. P.; Eur. J. Pharm. Sci. 2005, 26, 62.

20. De Montellano, P. R.; de Voss, J. J.; Nat. Prod. Rep. 2002, 19, 477.

21. Chorghade, M. S.; Hill, D. R.; Lee, E. C.; Pariza, R. J.; Pure Appl. Chem. 1996, 68,753 .

22. Maurin, A. J. M.; Iamamoto, Y.; Lopes, N. P.; Smith, J. R. L.; Bonato, P. S.; J. Braz. Chem. Soc. 2003, 14, 322.

23. Mansuy, D.; Battioni, P.; Battioni, J. P. Eur. J. Biochem. 1989, 184, 267.

24. Maurin, A. J. M.; Iamamoto, Y.; Maestrin, A. P. J.; Smith, J. R. L.; Santos, M. D.; Lopes, N. P.; Bonato, P. S.; J. Mol. Catal. A: Chem. 2005, 226, 23.

25. Sharefkin, J. G.; Saltzmann, H.; Org. Synth. 1963, 43, 62.

26. Merfort, I.; Phytochemistry 1992, 31, 2111.

27. Pauli, G. F.; Poetsch, F.; Nahrstedt, A.; Phytochem. Anal. 1998, 9, 177.

28. Carrier, M. N.; Scheer, C.; Gouvine, P.; Bertoli, J. F.; Battioni, P.; Mansuy, D.; Tetrahedron Lett. 1990, 31, 6645.

29. Artaud, I.; Ben-Aziza, K.; Mansuy, D.; J. Org. Chem. 1993, 58, 3373.

30. Tagliatesta, P.; Giovannetti, D.; Leoni, A.; Neves, M. G. P. M. S; Cavaleiro, J. A. S.; J. Mol. Catal. A: Chem. 2006, 252, 96.

31. Jin, X. H.; Ohgami, K.; Shiratori, K.; Suzuki, Y.; Koyama, Y.; Yoshida, K.; Ilieva, I.; Tanaka, T.; Onoe, K.; Ohno, S.; Exp. Eye Res. 2006, 82, 860 